\title{
ABORDAGEM DIAGNÓSTICA E TERAPÊUTICA DE HÉRNIA DIAFRAGMÁTICA PERITONEOPERICÁRDICA EM CÃO
}

(Diagnostic and therapeutic approach of peritoneaopericardial diaphragmatic hernia in the dog)

Brunna Silva Moreira ${ }^{1}$, Aline Medeiros Nakamura ${ }^{2}$, Aracelle Elisane Alves ${ }^{1}$, Francisco Claudio Dantas Mota $^{1}$

${ }^{1}$ Universidade Federal de Uberlândia, Faculdade de Medicina Veterinária; ${ }^{2}$ Programa de Pósgraduação em Cirurgia Veterinária FCAV/UNESP-Jaboticabal;

Corresponding author: brunna_moreira@hotmail.com

RESUMO: A hérnia peritoneopericárdica é uma má formação congênita incomum em cães e gatos, caracterizada por uma comunicação anômala entre o diafragma e o saco pericárdico, ocasionando a translocação das vísceras abdominais para o interior do saco pericárdico. Seu prognóstico é favorável e o tratamento mais indicado é o cirúrgico. Este relato teve como objetivo descrever o caso de um cão macho, da raça Shih Tzu, de 2 anos de idade com diagnóstico de hérnia diafragmática peritoneopericárdica compensada. Foi realizada a correção cirúrgica por meio da redução do conteúdo herniário, composto por fígado, baço, alças intestinais e omento, além da reconstituição simultânea do diafragma e pericárdio havendo resolução da anomalia congênita.

Palavras-chave: Canino; cirurgia; herniorrafia.

ABSTRACT: Peritoneopericardial is an uncommon congenital malformation in dogs and cats, characterized by anomalous communication between the diaphragm and the pericardial sac, causing the translocation of the abdominal viscera to the inside of the pericardial sac. Its prognosis is favorable and the most indicated treatment is the surgical. This paper aims at describing the diagnosis and treatment process in a dog with PPDH. This paper shows a 2-years-old male Shih Tzu diagnosed with compensated peritoneopericardial diaphragmatic hernia (PPDH). A reduction of the herniated content, composed of liver, spleen, intestinal loops and omentum, in addition to simultaneous reconstruction of the diaphragm and pericardium with resolution of the congenital anomaly.

Keywords: Dog; herniorrhaphy; surgery. 


\section{INTRODUÇÃO}

A hérnia diafragmática peritôniopericárdica (HDPP) é uma má formação congênita incomum em cães e gatos (Reimer et al., 2004), com baixa prevalência $(0,025 \%$ dos casos) (Burns et al., 2013), sendo o Weimaraner a raça mais predisposta entre os cães e pouco relatado na raça Shih Tzu (Burns et al., 2013; Reimer et al., 2004; Banz et al., 2010; Papazoglou et al., 2015).

A HDPP é decorrente de uma falha na embriogênese no momento da formação do septo transverso, que predispõe a uma comunicação anômala do diafragma ao saco pericárdico, possibilitando a entrada dos órgãos abdominais neste espaço (Liptak et al., 2002; Debiak et al., 2009). A maioria dos animais que sofrem essa alteração são diagnosticados nos primeiros anos de vida, concomitantes a outras alterações congênitas, tais como hérnia umbilical na porção cranial do abdômen, malformação no esterno e cardiopatias (Burns et al., 2013).

Os sinais clínicos são inespecíficos, geralmente associados ao desencadeamento de sintomatologia clínica de ordem respiratória ou gastrointestinal como dispneia, tosse, respiração ofegante, anorexia, êmese, diarreia, entre outros (Burns et al., 2013; Kheirandish et al., 2014).

A passagem de órgãos abdominais para o saco pericárdico pode resultar em tamponamento cardíaco, obstrução ou encarceramento dos órgãos herniados (Burns et al., 2013). Contudo, existem relatos de caso de animais assintomáticos com idade avançada por não existir comprometimento dos órgãos herniados, com diagnóstico incidental confirmado por radiografias torácicas ou necropsia (Cunha et al., 2000; Quintavalla e Zannetti, 1998; Kheirandish et al., 2014).

O diagnóstico baseia-se através do exame radiográfico, com qual se denota a presença de vísceras abdominais no interior do saco pericárdico, perca da distinção entre o coração e diafragma, além de aumento da silhueta cardíaca. Demais exames complementares de imagens representam importante ferramenta no diagnóstico da HDPP, tais como radiografia contrastada do trato gastrointestinal, ultrassonografia, ecocardiograma e tomografia computadorizada são utilizados na distinção de anomalias congênitas associadas (Reimer et al., 2004; Banz e Gottfried, 2010; Burns et al., 2013).

O tratamento conservador é utilizado em pacientes idosos e assintomáticos, em virtude das possíveis aderências em estruturas herniadas, sendo o procedimento cirúrgico nestes casos contraproducente por lesionar as estruturas envolvidas (Bellah et al., 1989; Reimer et al., 2004).

O tratamento cirúrgico na maioria das vezes é a melhor opção, com prognóstico favorável mediante diagnóstico precoce (Benlloch-gonzalez e Poncet, 2015). Consiste na redução do conteúdo herniário para o seu local de origem e a reconstrução das estruturas anômalas com a herniorrafia, de modo a evitar complicações e quadros de descompensação aguda (White et al., 2003; Burns et al., 2013).

Objetivou-se com este trabalho descrever um caso de hérnia peritoneopericárdica compensado em um cão de dois anos de idade submetido a herniorrafia.

\section{DESCRIÇÃO DO CASO}

Um canino da raça Shih Tzu de dois anos de idade, $4,5 \mathrm{~kg}$ foi atendido no Hospital Veterinário da Universidade Federal de Uberlândia, para correção de hérnia umbilical. $O$ animal apresentava histórico de vômitos esporádicos, principalmente após refeições ou exercícios físicos desde filhote. Tutor relatou tentativa de herniorrafia umbilical em outra clínica, mais que em virtude de 
complicações anestésicas não foi realizada.

Durante o exame físico observouse mucosas normocoradas, frequência cardíaca de 80 batimentos por minuto, frequência respiratória de 24 movimentos respiratórios por minuto, temperatura corporal de $38{ }^{\circ} \mathrm{C}$ e normohidratação. $\mathrm{Na}$ auscultação constatou-se abafamento de bulhas cardíacas e na palpação presença de choque de ponta pouco evidente. Os exames laboratoriais de hemograma, perfil renal e hepático encontravam-se dentro dos valores de referência para a espécie.

A radiografia simples da região torácica denotou elevação dorsal da traqueia, aumento da silhueta cardíaca, além de discretas estruturas de conteúdo gasoso em seu interior, próximo às margens cardíaca $\mathrm{e}$ diafragmática (Figura 1A). $\mathrm{O}$ exame radiográfico contrastado com sulfato de bário do sistema gastrointestinal evidenciou a presença de alças intestinais ao redor da sombra cardíaca, confirmando a hérnia peritoneopericárdica (Figura 1B e 1C).

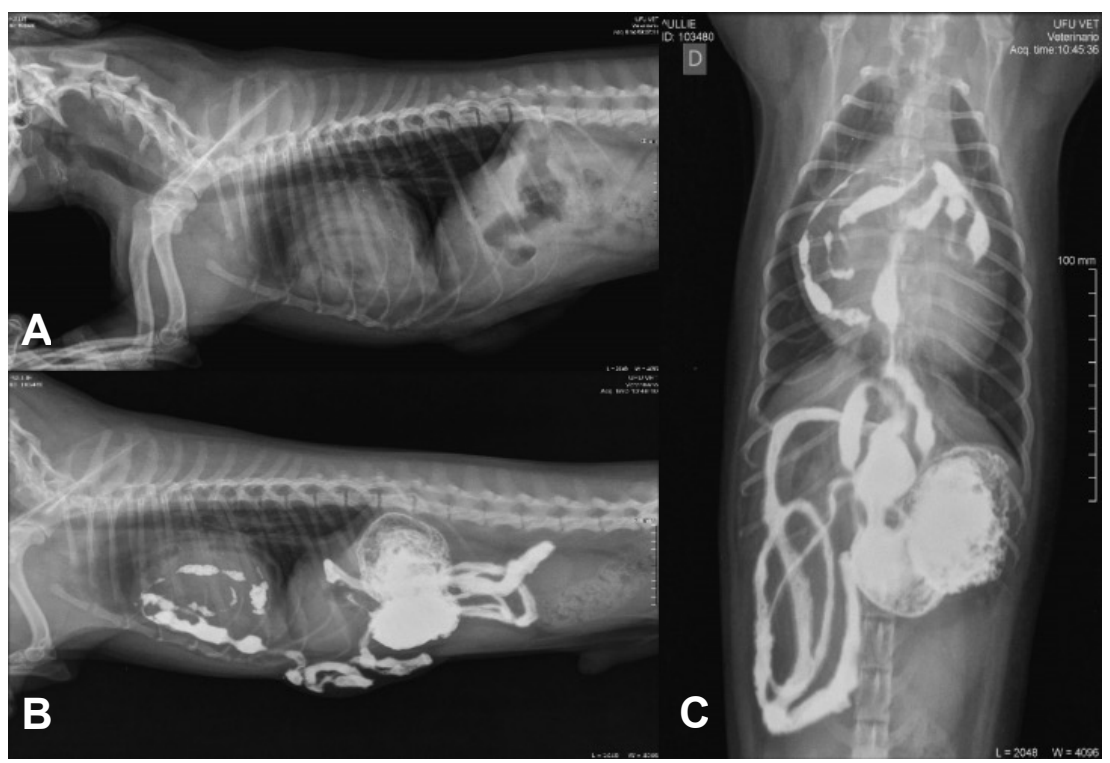

Figura 1. Imagens radiográficas da região toracoabdominal de cão acometido com hérnia diafragmática peritoneopericárdica. [A] Radiografia simples na projeção lateral, com qual nota-se elevação dorsal da traqueia e aumento da silhueta cardíaca com a presença de conteúdo gasoso em seu interior. [B e C] Imagem contrastada (sulfato de bário) na posição látero-lateral e ventro-dorsal, observa-se a presença do contraste (aumento da radiopacidade no saco pericárdico) em alças intestinais, dispostas na cavidade torácica ao redor da sombra cardíaca.

O exame ecocardiográfico foi realizado como exame pré-operatório e para reconhecimento do conteúdo herniado no saco pericárdico, com qual destacou-se a presença de alças intestinais intrapericárdicas, porém sem a presença de alterações cardíacas e hemodinâmicas. $\mathrm{O}$ paciente foi encaminhado para a cirurgia para correção da anomalia.
Após preparo de rotina da área cirúrgica, foi realizado incisão de pele e subcutâneo, na região mediana, sobre a hérnia umbilical, identificando o omento e alças intestinais, como conteúdo do saco herniário. Dessa forma, parte das alças intestinais foram expostas de forma a melhorar a visualização do defeito diafragmático.

O defeito diafragmático 
encontrava-se dorsolateralmente ao processo xifóide, comunicando a cavidade abdominal ao saco pericárdico. Estavam herniados no saco pericárdico partes do intestino delgado, omento, baço e o lobo hepático medial direito (Figura 2), a redução foi realizada de forma cuidadosa.

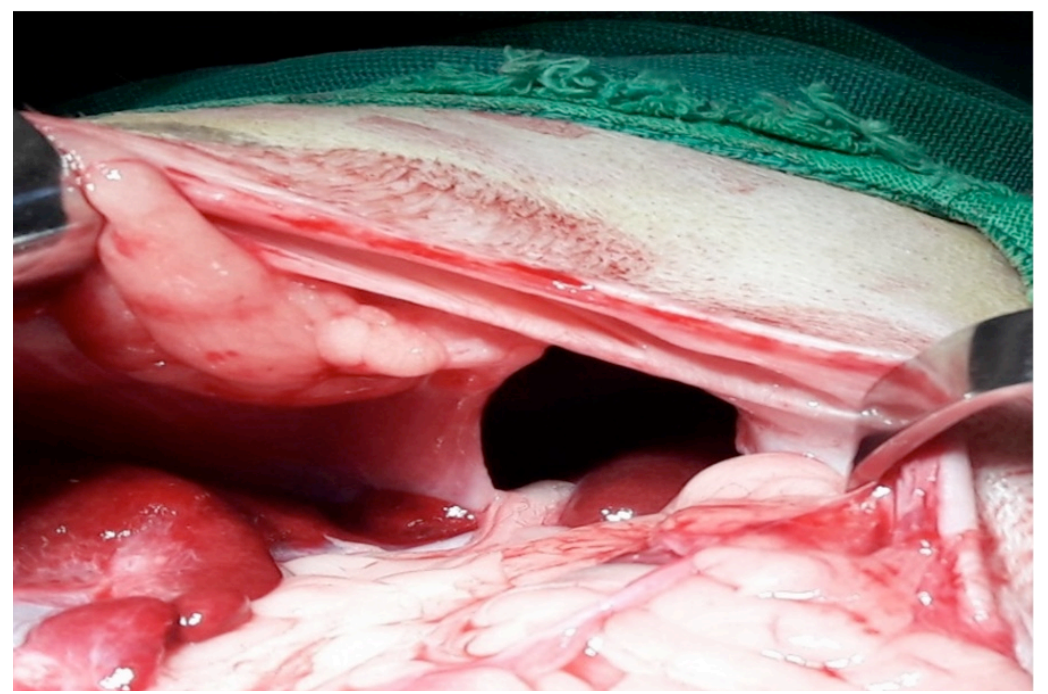

Figura 2. Exposição primária do anel (seta amarela) e conteúdo herniário. Nota-se o avanço do parcial do baço (seta vermelha) e omento para a cavidade torácica.

As bordas ao redor das hérnias, diafragmática e umbilical, foram desbridadas e ambas aproximadas com sutura em padrão Sultan, com fio de

mononylon 2-0 (Figura 3). A síntese do diafragma promoveu a oclusão simultânea do saco pericárdico pelo fato das duas estruturas estarem fundidas.

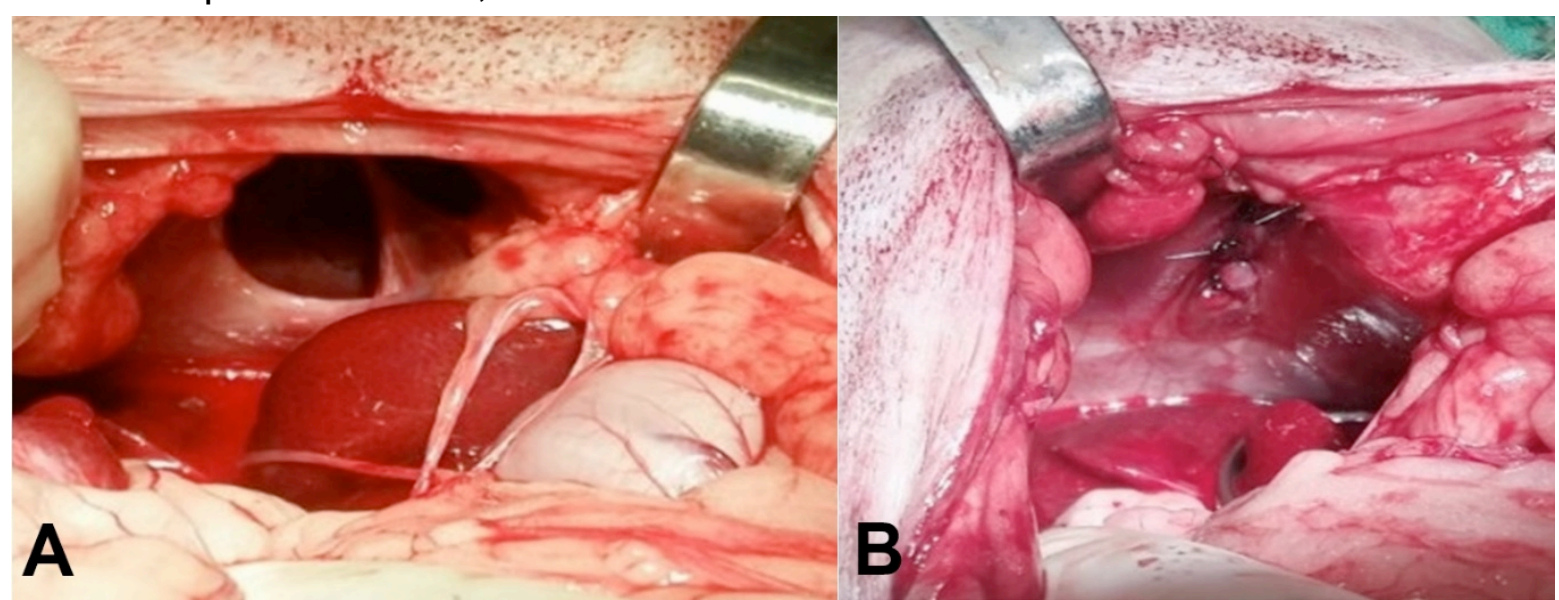

Figura 3 - Nota-se em A: exposição completa do anel herniário, após reposicionamento cuidadoso dos órgãos abdominais, além da fusão do saco pericárdico no bordo diafragmático (seta azul). Nota-se em B: aspecto final da reconstrução da musculatura do diafragma após síntese.

Devido ao grande defeito do músculo reto do abdome decorrente da hérnia umbilical, bem como a pequena complacência da cavidade abdominal, não foi possível o retorno das vísceras herniadas e o fechamento da mesma. Neste momento, optou se pela esplenectomia total e a remoção de parte do omento, o que permitiu a síntese do músculo reto abdominal com pontos em padrão Sultan e aproximação do subcutâneo em padrão zig-zag, ambos com fio de ácido poliglicólico 3-0. A dermorrafia deu-se com fio mononylon 3-0, utilizando padrão Wolf. 


\section{DISCUSSÃO}

A maioria dos pacientes são diagnosticados ainda juvenis. Entretanto, há relatos de diagnósticos em animais adultos, como no presente relato (Quintavalla e Zannetti,1998; Reimer et al., 2004; Banz e Gottfried, 2010; Kheirandish et al., 2014). Hérnias peritoneopericárdicas podem ser assintomáticas quando não há comprometimento das vísceras, porém animais com hérnia supraumbilical, umbilical ou com criptorquidismo, devem ser investigados quanto à presença de sinais condizentes com desconforto respiratório ou alterações gastrointestinais, com qual preconiza-se a realização de exames complementares que evidencie a presença DHPP (Burns et al., 2013).

Os sinais clínicos foram compatíveis com outros estudos, onde se observou tosse, diminuição dos sons pulmonares e abafamento dos sons cardíacos na ausculta, indistinção da localização do choque de ponta à palpação, abdômen fino e anomalia congênita associada (hérnia umbilical do abdome cranial) (Banz e Gottfried, 2010; Burns et al., 2013). Nestes casos acredita-se que as tosses sejam causadas pela compressão do brônquio principal, secundário ao aumento da silhueta cardíaca (Liptak et al., 2002) e o abafamento dos sons cardíacos, diminuição dos sons pulmonares e dificuldade de reconhecimento do choque de ponta deve-se à presença de conteúdo herniado ocupando o espaço no saco pericárdico (Banz e Gottfried, 2010).

No presente relato optou-se pelos exames de radiografia e ecodopplercardiograma, pois segundo Debiak et al. (2009) são exames essenciais para o estabelecimento do diagnóstico de hérnia diafragmática peritoneopericárdica. Sendo assim, os sinais radiográficos de uma HDPP incluem o aumento da silhueta cardíaca, presença de conteúdo herniado na cavidade torácica delimitada pela sombra cardíaca e impossibilidade de delimitar a continuidade da borda diafragmática, devendo ser distinguida de derrame pleural ou aumento do coração. Por vezes, apenas a presença de alças intestinais repleta de gás e conteúdo ao redor da sombra cardíaca pode indicar fortemente o diagnóstico de HDPP (Cunha et al., 2000; Cariou et al., 2009) assim como observado no presente relato.

A radiografia torácica simples possibilita o diagnóstico de HDPP, porém quando associada a outros meios diagnósticos com a radiografia contrastada à base de bário, ultrassonografia abdominal, ecocardiograma e a tomografia computadorizada auxiliam na detecção de quaisquer anomalias congênitas associadas (Wallace et al., 1992; Reimer et al., 2004; Banz e Gottfried, 2010; Burns et al.,2013).

O exame ecocardiográfico é indicado e fidedigno para confirmação do diagnóstico, pois oferece conhecimento relativo das estruturas herniadas e avaliação clínica da função cardíaca (Quintavalla e Zannetti,1998), mediante sua associação aos demais exames de imagem foi possível aumentar a sensibilidade diagnóstica e foi imprescindível para o planejamento cirúrgico.

Para o tratamento do paciente optou-se pela cirurgia de herniorrafia, considerada de grande eficácia para solucionar o quadro clínico. O saco pericárdico estava distendido e comunicando-se com a cavidade abdominal e no seu interior estavam presentes omento, alças intestinais e um lobo hepático, o que é de incidência comum em casos de HDPP (Reimer et al., 2004; Banz e Gottfried, 2010).

A correção do defeito herniário foi precedida pela aposição primária das margens diafragmáticas, através da 
utilização de um único padrão de sutura interrompido, com material inabsorvível monofilamentoso, conforme proposto por Burns et al. (2013) e Banz e Gottfried (2010).

Porém, em alguns casos quando há a presença de grandes defeitos e aderências entre os órgãos herniados, com quais não possibilitam a realização do fechamento primário deverá ser avaliado a necessidade da facilitação do acesso cirúrgico por meio de esternotomia mediana caudal, além da realização de enxertos pericárdicos, flapes musculares e a utilização de malhas sintéticas à base de polipropileno para a oclusão herniária completa (Burns et al., 2013; McClaran, 2013).

Segundo Dörfelt et al. (2012), o volume excessivo das vísceras abdominais associado a condições que limitem a expansão da parede abdominal, são fatores que contribuem para aumento da pressão intraabdominal (PIA). Este aumento em longo prazo gera falhas orgânicas e cria um quadro clínico denominado de síndrome da compartimentalização, representada por efeitos deletérios cardiovasculares, pulmonares, renais, gastrointestinais e hepáticos em resposta a alterações de diminuição do fluxo sanguíneo e débito cardíaco. Sendo este um dos fatores que leva a indicação da correção precoce da HDPP, enquanto o animal ainda está em crescimento (Bellah et al., 1989).

No presente relato, por se tratar de um caso crônico, resultando na baixa complacência da cavidade abdominal, foi necessário a esplenectomia total e remoção parcial do omento para que a síntese da parede abdominal pudesse ser executada sem o desenvolvimento da PIA. Pachter et al. (1998), considerou a realização da esplenectomia e retirada parcial do omento, como métodos auxiliares na restauração da integridade da cavidade abdominal sem promover o aumento considerado da PIA, além disso a incidência de infecções secundárias decorrentes da retirada do baço é incomum em cães e gatos, quando comparado aos humanos.

Como complicações pósoperatória em cães destacam-se a deiscência incisional, pericardite ou pneumotórax. $O$ fornecimento de oxigênio é necessário para evitar complicações respiratórias secundárias (Wallace et al., 1992; Banz e Gottfried, 2010; Burns et al., 2013), porém no caso em questão não houve nenhuma complicação pós-operatória.

\section{CONCLUSÃO}

O estudo de exames de imagem, tais como o ecodopplercardiograma e radiografia contrastada foram imprescindíveis para o diagnóstico final. A correção cirúrgica mostrou-se efetiva no presente relato, sem apresentar complicações.

\section{REFERÊNCIAS}

BANZ, A. C.; GOTTFRIED, S. D. Peritoneopericardial diaphragmatic hernia: a retrospective study of 31 cats and eight dogs. Journal of the American Animal Hospital Association, v.46, p.398-404, 2010.

BELLAH, J. R; WHITTON, D. L.; ELLISON, G. W.; PHILLIPS, L. Surgical correction of concomitant cranioventral abdominal wall, caudal sternal, diaphragmatic, and pericardial defects in young dogs. Journal the American Veterinary Medical Association, v.195, n.12, p.1722-1726, 1989.

BENLLOCH-GONZALEZ, M.; PONCET, C. Sternal cleft associated with Cantrell's pentalogy in a German shepherd dog. Journal the American Animal Hospital Association, v.51, p.279-284, 2015.

BURNS, C. G.; BERGH, M. S.; 
MCLOUGHLIN, M. A. Surgical and nonsurgical treatment of peritoneopericardial diaphragmatic hernia in dogs and cats: 58 cases (19992008). Journal of the American Veterinary Medical Association, v.242, p.5, p.643-550, 2013.

CARIOU, M. P. L., KENNY, P.; BAINES, S. J. B. Surgical management of an incidentally diagnosed true pleuroperitoneal hernia in a cat. Journal of feline medicine and surgery, v.11, n.10, p.873-877, 2009.

CUNHA, O.; DA FILHO, S. T. L. P.; BARBOSA, A. G. R.; PORTELLA, L. C. V. Hérnia peritoneopericárdica em cão. Ciência Rural, v.30, n.5, p.899-902, 2000.

\begin{tabular}{|c|c|}
\hline $\begin{array}{l}\text { DEBIAK, } \\
\text { PZCEPANIAK, } \\
\text { Diagnostics } \\
\text { jeritonealpericardial } \\
\text { lérnia } \\
\text { (PPDH). }\end{array}$ & $\begin{array}{r}\text { LOJSZCZYK- } \\
\text { KOMSTA, R. } \\
\text { canine } \\
\text { diaphraamatic }\end{array}$ \\
\hline & \\
\hline
\end{tabular}

DÖRFELT, R.; AMBRISKO, T.D.; MOENS, Y. Influence of fentanyl on intra-abdominal pressure during laparoscopy in dogs. Veterinary Anaesthesia and Analgesia, v.39, p.390-397, 2012.

KHEIRANDISH, R.; SABERI, M.; VOSOUGH, D.; ASKARI, N. Congenital peritoneopericardial diaphragmatic hernia in a terrier dog. Veterinary Research Forum, v.5, n.2, p.153-155, 2014.

LIPTAK,J. M.; BISSET, S. A.; ALLAN, G. S.; ZAKI S. \& MALIK R. Hepatic cysts incarcerated in a peritoneopericardial diaphragmatic hernia. Journal of Feline Medicine and Surgery, v.4, n.2, p.123125, 2002.

QUINTAVALLA, C.; ZANNETTI, G. A case of diaphragmatic peritoneal pericardial hernia in a dog. Universi tà degl i Studi di Parma - Annal i del la
Facol tà di Medicina Veterinária, $\mathrm{Vol}$. XVI II . 1998.

MC CLARAN, L.K. Diaphragmatic and peritoneopericardial diaphragmatic hernias. In: (ed: Monnet E) Small Animal Soft Tissue Surgery. WilleyBlackwell, Ames, p. 278-285, 2013.

PACHTER, H. L.; GUTH, A.; HOFSTETTER, S. R. Changing patterns in the management of splenic trauma: the impact of nonoperative management. Annals of Surgery, v.227, p.708, 1998.

PAPAZOGLOU, L.G.; PATSIKAS, M.N.; DELIGIANNI, A.; WISNER, E.R.; KAZAKOS G. Pneumopericardium associated with peritoneopericardial diaphragmatic hernia repair in a dog.Veterinary Medicine, v.110, n.4., p.94-98, 2015.

REIMER, S. B.; KYLES, A. E.; FILIPOWICS, D. E.; GREGORY, C. R. Long-term outcome of cats treated conservatively or surgically for peritoneopericardial diaphragmatic hernia: 66 cases (1987-2002). Journal of the American Veterinary Medical Association, v.224, n.5, p.728-732, 2004.

WALLACE, J.; MULLEN, H.S.; LESSER, M.B. A Technique for surgical correction of peritoneal pericardial diaphragmatic hernia in dogs and cats. Journal of the American Animal Hospital Association. V.28, p.503-510, 1992.

WHITE, J. D.; TISDALL, P. L. C.; NORRIS, J. M.; MALIK, R. Diaphragmatic hernia in a cat mimicking a pulmonary mass. Journal of Feline Medicine and Surgery, v.5 n.3, p.197201, 2003. 